Check for updates

The BMJ

Cite this as: BMJ2020;371:m4709 http://dx.doi.org/10.1136/bmj.m4709 Published: 02 December 2020

\section{Covid-19: Moderna applies for US and EU approval as vaccine trial reports $94.1 \%$ efficacy}

\author{
Elisabeth Mahase
}

The drug company Moderna has applied for emergency use authorisation from the US Food and Drug Administration and conditional approval from the European Medicines Agency for its covid-19 vaccine.

The move comes after new results were released from its phase III trial, showing vaccine efficacy of $94.1 \%$ based on 196 covid-19 cases, of which 185 were in the placebo group.

The company expects an FDA meeting to take place on 17 December to review the safety and efficacy data. It also plans to submit the phase III data to a peer reviewed publication.

By the end of 2020 an estimated 20 million doses of the vaccine should be available in the US, and 500 million to a billion doses are expected worldwide in 2021. The EU has secured a deal for 80 million to 160 million doses, while seven million doses have been separately agreed for the UK.

The randomised, 1:1 placebo controlled study included 30000 adults in the US and was designed in collaboration with the FDA and the National Institutes of Health. It tested the mRNA-1273 vaccine, with participants receiving two $100 \mu \mathrm{g}$ doses one month apart. ${ }^{1}$ Moderna had previously (16 November) reported interim data from the trial based on 95 cases, which estimated efficacy at $94.5 \% .^{2}$

\section{Severity and safety}

One of the trial's secondary endpoints analysed severe covid-19 cases, of which there were 30 . All severe cases occurred in the placebo group. One covid-19-related death has occurred in the study to date, also in the placebo group.

In the press release announcing the data Moderna said that efficacy was "consistent across age, race and ethnicity, and gender demographics.” Of the 196 covid-19 cases, 33 were in people aged over 65, and 42 were in participants identified as being from diverse communities (29 Hispanic or LatinX, six Black or African Americans, four Asian Americans, and three multiracial).

Regarding safety, Moderna said that a continuous review was ongoing but that no serious safety concerns had been identified. The most common solicited adverse reactions were injection site pain, fatigue, myalgia, arthralgia, headache, and erythema/redness at the injection site. The frequency and severity of these reactions increased in the mRNA-1273 group after the second dose.

Stephen Evans, professor of pharmacoepidemiology at the London School of Hygiene \& Tropical Medicine, said, "While the best estimate is $94.1 \%$ against all covid-19 disease, the statistical uncertainty in this is such that the data are compatible with a true efficacy of about $87 \%$.

"This is of course, still a very good efficacy. Similarly, the $100 \%$ efficacy against more severe disease is compatible with an efficacy of $90 \%$-again, this is very good and is some evidence that severe as well as mild disease is prevented."

Azra Ghani, infectious disease epidemiology chair at Imperial College London, said, "The results have been tested across a diverse population and are reported as being consistent in different subgroups, although these numbers are not given and we should wait for further information in the scientific article that is being prepared.

“Although not yet reported, the trial includes a secondary endpoint of asymptomatic infection: efficacy against this would be very welcome, as it would give the first indication of the broader indirect impact that widespread vaccination could have in reducing onward spread."

\section{Moderna. Clinical study protocol. 20 Aug 2020. https://www.moder- natx.com/sites/default/files/mRNA-1273-P301-Protocol.pdf. \\ 2 Mahase E. Covid-19: Moderna vaccine is nearly 95\% effective, trial involving high risk and elderly people shows. BMJ 2020;371:m4471doi: 10.1136/bmi.m4471.}

This article is made freely available for use in accordance with BM|'s website terms and conditions for the duration of the covid-19 pandemic or until otherwise determined by BMJ. You may use, download and print the article for any lawful, non-commercial purpose (including text and data mining) provided that all copyright notices and trade marks are retained. 